\title{
Surgical site infection: clean surgery and antimicrobials
}

\author{
Yusuke Asakura $^{1}\left[\right.$ Daho Kinoshita ${ }^{2} \cdot$ Makoto Ozaki $^{2}$
}

Received: 24 February 2017 / Accepted: 25 March 2017 / Published online: 4 April 2017

(C) Japanese Society of Anesthesiologists 2017

\section{To the Editor:}

According to the guideline for antimicrobial prophylaxis for surgical site infection (SSI), cefazolin (CEZ) is the drug of choice in most surgical procedures [1]. The predominant microbial organism that causes SSI after clean surgery is Staphylococcus aureus, accounting for $22.5 \%$ of SSIs. We evaluated the antimicrobial susceptibility profile of methicillin-sensitive $S$. aureus (MSSA) in a tertiary care hospital in Japan.

The study was conducted in a retrospective fashion after IRB approval was obtained. The samples consisted of 695 MSSA isolates from 533 individuals during the 4-year period from 2012-2015. Minimal inhibitory concentrations (MICs) for CEZ as well as the antimicrobials shown in Supplementary Fig. 1 were determined according to the Clinical and Laboratory Standards Institute guidelines. Of 695 MSSA isolates, no single resistance to CEZ as well as flomoxef and imipenem was observed (Supplementary Fig. 1). Clindamycin showed a high resistance rate (129 out of 695 isolates were resistant; 18.5\%), suggesting that it may not be suitable for an antimicrobial agent for patients with a known history of $\beta$-lactam allergy. Although sensitivity to vancomycin was well preserved, 2 out of 695 isolates have been identified as vancomycin insensitive (MIC $>4-8 \mathrm{mg} / \mathrm{L}$ ), supporting the well-known notion that vancomycin is less effective than CEZ for MSSA. Resistance to fluoroquinolones and aminoglycosides (except for arbekacin) was also high. The results including the sensitivities of other antimicrobials are summarized in Supplementary Fig. 1.

\section{Reference}

1. Bratzler DW, Dellinger EP, Olsen KM, Peri TM, Auwaerter PG, Bolon MK, Fish DN, Napolitano LM, Sawyer RG, Slain D, Steinberg JP, Weinstein RA. Clinical practice guidelines for antimicrobial prophylaxis in surgery. Am J Health Syst Pharm. 2013;70:195-283.
Electronic supplementary material The online version of this article (doi:10.1007/s00540-017-2348-9) contains supplementary material, which is available to authorized users.

Yusuke Asakura

yasakura@kaikou.or.jp

1 Departments of Anesthesiology, Nagoya Kyoritsu Hospital, Hokke, Nakagawa-ku, 1-172 Nagoya, Aichi, Japan

2 Tokyo Womens' Medical University, Tokyo, Japan 